\title{
The prevalence and distribution of human papillomavirus among 10,867 Chinese Han women
}

\author{
Chunlei Guo ${ }^{1,2+}$, Hui Du ${ }^{1,2+}$, Jerome L. Belinson ${ }^{3}$, Chun Wang ${ }^{1,2}$, Xia Huang ${ }^{1,2}$, Xinfeng Qu ${ }^{4}$, Ruifang Wu ${ }^{1,2^{*}}$ (D) \\ CHIMUST team
}

\begin{abstract}
Objective: To assess the prevalence and distribution of HPV genotypes among Chinese Han women, and to explore the risk of high-grade cervical lesions associated with individual hr-HPV genotypes.
\end{abstract}

Methods: Genotyping and histopathology data from the Chinese Multi-Center Screening Trial (CHIMUST) and its pilot screening trial, from 6 regions across mainland China, were re-analyzed. The data from physician- and selfcollected samples from 10,867 Chinese Han women (ages 30-69) were used to determine the prevalence and distribution of hr-HPV and to explore the risk association between hr-HPV genotypes and precancerous lesions.

Results: $9.2 \%$ of the study population tested hr-HPV positive in physician-collected sample. The prevalence varied regionally from the lowest in Guangdong (6.3\%) to the highest in Inner Mongolia (13.0\%). The most prevalent genotypes found were HPV-52 (21.7\%), HPV-16 (19.2\%), HPV-58 (15.0\%), HPV-39 (8.9\%), and HPV-51 (8.2\%). The overall odds ratios for CIN2+ and CIN3+ for the presence of HPV-16 was $58.6(95 \% \mathrm{Cl} 39.2-87.5)$ and, $91.6(95 \% \mathrm{Cl}$ 54.3-154.6), respectively and remained the highest odds ratio for CIN3+ in all 6 regions.

Conclusion: Geographical variation exists in the prevalence and distribution of hr-HPV in mainland China. HPV-16/ 52/58 were the most prevalent genotypes, and HPV-16 had the highest risk for high-grade cervical lesions.

Trial registration: CHIMUST, Registration number: ChiCTR-EOC-16008456. Registered 11 May 2016.

Keywords: Human papillomavirus, Type-specific prevalence, Distribution, risk, Chinese Han

\section{Introduction}

Cervical cancer is ranked as the fourth most common cancer in women, with an estimated of 570,000 newly diagnosed cases and over 310,000 deaths in 2018 worldwide, more than three quarter of these are from low-income and middle-income countries (LMICs) [1]. China, as well as India, is one of the top contributors

\footnotetext{
* Correspondence: wurf100@126.com

${ }^{\dagger}$ Chunlei Guo and Hui Du contributed equally to this work.

'Department of Obstetrics and Gynecology, Peking University Shenzhen Hospital, No. 1120, Lianhua Road, Shenzhen, Guangdong 518036, PR China

${ }^{2}$ Shenzhen Key Laboratory on Technology for Early Diagnosis of Major Gynecological Diseases, Shenzhen, Guangdong, PR China

Full list of author information is available at the end of the article
}

to the global burden of cervical cancer, newly diagnosed cases have exceeded 100,000 and caused nearly 50,000 deaths per year [2].

Persistent high-risk human papillomavirus (hr-HPV) infection by certain types is the main cause of cervical cancer and cervical intraepithelial neoplasia [3]. HPV is a small $(8 \mathrm{~kb})$, non-enveloped, and double stranded circular DNA virus with tropism for cutaneous and mucosal epithelial cells. After infection, the virus prevents cellular apoptosis by degrading p53 which contributes to the accumulation of genetic mutations. This leads to immortalization through viral protein E6 and stimulates cell proliferation by degrading the 
retinoblastoma tumor suppressor gene $(\mathrm{Rb})$ which in turn stimulates host genome instability through viral protein E7 [4]. Thus far, more than 200 papillomavirus genotypes have been identified and 25 of these are oncogenic to humans. At least 14 genotypes (HPV-16、-18、-31、33、-35、-39、-45、-51、-52、-56、-58、-59、-66 and -68) have been classified into hr-HPV due to their capability to cause cervical cancer and cervical intraepithelial neoplasia [5]. However, the carcinogenic potential varies greatly by genotype; HPV-16 and -18 together are reported to be responsible globally for $71 \%$ of cervical cancer [6]. Moreover, a significantly geographical variation exists in prevalence and type-distribution of HPV infection [7]. Since HPV vaccination and primary HPV screening are considered two powerful weapons against cervical cancer, epidemiological knowledge of the prevalence and distribution of cervical HPV infection in the general population becomes critical. In this study, the data from the Chinese Multi-Center Screening Trial (CHIMUST) and the Shenzhen Pilot Screening Trial which together enrolled 11, 143 women from 6 regions in northern, central and southern mainland China, were re-analyzed to assess the prevalence and distribution of HPV genotypes among Chinese Han women, and to explore the risk of high-grade cervical lesions associated with individual hr-HPV genotypes.

\section{Materials and methods \\ Study population}

This Chinese Multi-Center Screening Trial (CHIMUST) (Registration number: ChiCTR-EOC-16008456) is a multi-center, cross-sectional, population-based cervical cancer screening trial conducted between Aug 2016 and Jan 2018 in 5 regions in mainland China: Inner Mongolia (Northern China), Hebei province (Northern China), Hubei province (Central China), Jiangxi province (Central China) and Guangdong province (Southern China). Women who were non-pregnant, sex exposed, no prior pelvic radiation, no hysterectomy, and no cervical cancer screening in the past 3 years were eligible to be enrolled. The protocol of this trial was approved by the Institutional Review Board (IRB) of Peking University Shenzhen Hospital (IRB: PUSH2016001) and Cleveland Clinic Institutional Review Board (IRB:15-1549). Before CHIMUST, in 2014 our team conducted a Pilot Screening Trial in Shenzhen city using the same protocol. A total of 12,897 women between 30 and 69 years of age were enrolled in these two screening trials, but only 10,867 Han ethnicity women were included in this study. Of these 10,867 Han ethnicity women, 8891 were from CHIMUST while the other 1976 were from Shenzhen Pilot Screening Trial. All participants signed an informed consent document before enrollment.
All participants provided a self-collected vaginal sample and a physician-collected endocervical sample. The physician-sampling was performed following selfsampling. The physician placed a vaginal speculum to expose the cervix, then obtained a cervical exfoliated cell sample at the squamocolumnar junction of the cervix. All samples were tested with the PCR-based high-risk HPV assays: Cobas and SeqHPV (BGI, Shenzhen, China). The physician-collected samples were also processed and interpreted by PUSH cyto-pathologists using the Hologic I2 Imager (computer assisted cytology). Women testing HPV positive by either Cobas or SeqHPV (self or direct) were referred for colposcopy, using the quadrant-based POI (Preventive Oncology International Inc.) protocol of directed and random biopsies plus ECC [8]. The implementation and methods of the trial have been detailed in prior publications $[9,10]$.

For this manuscript, we merged the data from the Shenzhen Pilot Screening Trial and CHIMUST, and made the following revisions: a. Shenzhen is listed as a new first-tier city as its population is dominated by immigrants; b. The Mentougou District of Beijing is actually located in the administrative area of Hebei Province, so these two parts of data were merged. Finally, listing 6 screening centers: 1. Inner Mongolia; 2. Hebei province; 3. Hubei province; 4. Jiangxi province; 5 . Guangdong province; 6 . Shenzhen city. Of these 6 screening centers, Inner Mongolia and Hebei province located in northern mainland China, Hubei province and Jiangxi province located in central mainland China, Guangdong province and Shenzhen city located in southern mainland China. The data referring to HPV genotyping of physician-collected and self-collected samples with SeqHPV and the associated histologic diagnoses among Chinese Han women were reanalyzed for this manuscript (see Fig. 1).

\section{Study methods \\ SeqHPV assay}

The SeqHPV is a PCR assay for high-risk HPV based on next-generation sequencing (NGS). It was developed by BGI Shenzhen, P.R. China. This NGS assay uses indexmultiplex-PCR techniques to amplify the HPV L1 gene of cervical exfoliated cells in the sample using a "double index system". It is a high-throughput technology that can identify 14h-HPV genotypes (HPV-16、-18、$31 、-33 、-35 、-39 、-45 、-51 、-52 、-56 、-58 、-59 、-$ 66 and -68) [11]. All procedures were carried out in strict accordance with the working manual of the testing technology and the guidelines for the companion kit.

\section{Pathological diagnosis of colposcopic biopsy}

Women testing HPV positive for any HPV sample (self or direct on either assay) were called back for 


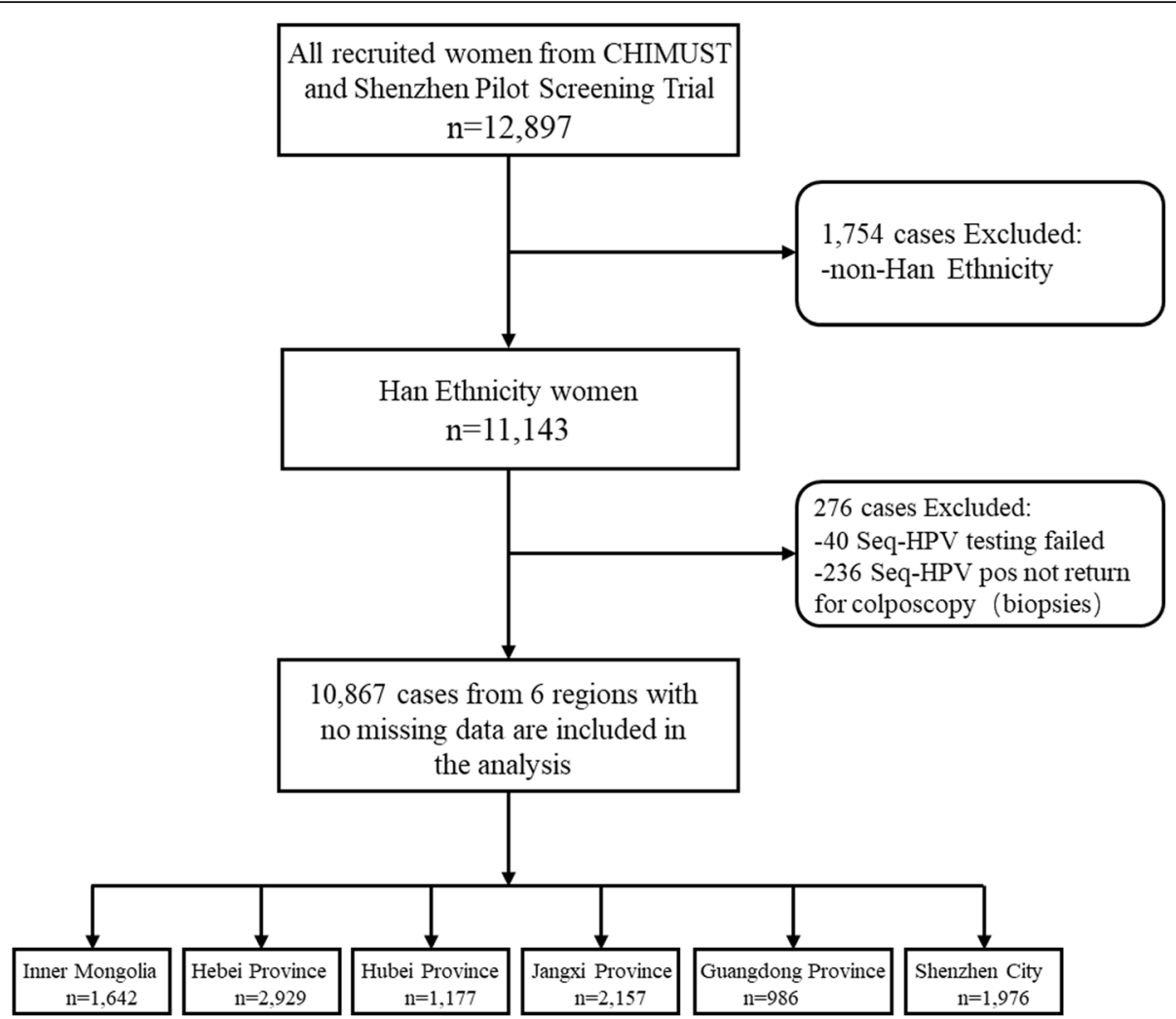

Fig. 1 Study population

colposcopy. The colposcopy protocol used in this trial was done by quadrant. We used directed biopsies for all visible lesions, and random biopsies at the squamocolumnar junction in normal quadrants. All colposcopy patients had an endocervical curettage (ECC) [8]. Study colposcopists and pathologists were blinded as to the cytology results. Pathological diagnoses included negative (for intraepithelial lesion/malignancy), cervical intraepithelial neoplasia 1 (CIN1), CIN2, CIN3, microinvasive cancer, and invasive cancer. The highest grade among the multiple biopsies from each quadrant and the ECC was recorded as the final diagnosis.

\section{Statistical analysis}

SPSS v.24.0 software (IBM, Armonk, NY, USA) was used for all data analysis in this study. Odds ratios and 95\% confidence intervals $(95 \% \mathrm{CI})$ were calculated by univariate logistic regression modelling to assess the risk of CIN2+/CIN3+ associated with hr-HPV genotypes. McNemar's Chi-square was performed to calculate differences between paired proportions at a probability level of 0.05. Agreement between physician- and self-collected sample was measured by absolute agreement and Kappa statistics (Cohen's Kappa).

\section{Results}

\section{General results}

A total of 12,897 women from 6 regions were recruited in CHIMUST and its pilot screening trial. Of these, 1754 of non-Han ethnicity, 40 Seq HPV testing failures and 236 Seq HPV positives who did not return for colposcopy were excluded. Therefore a total of 10,867 Chinese Han women with mean age $44.5 \pm 7.6$ years had complete data are included in this analysis. Among the 10,867 Chinese Han women, 10,508 (96.7\%) were pathologically normal, 242 (2.2\%) had CIN1, 116 (1.1\%) had CIN2+ and 62 (0.6\%) had CIN3 + .

\section{Concordance of the Seq HPV Assays in Physician- collected and Self-collected Sample}

A total of 995(9.2\%) women were positive for Seq HPV with specific HPV genotypes in physician-collected sample while 1030(9.5\%)in self-collected sample. And 912 women tested positive for Seq HPV in both two sampling methods. The concordance rates of HPV positive between physician-collected and self-collected sample was $81.9 \%(912 / 1113)$. The agreement in Seq HPV detection between physician- and self-collected sample was very $\operatorname{good}(\mathrm{Kappa}=0.89,95 \%$ CI $0.88-0.91$, $P<0.001)$. 


\section{Type-specific hr-HPV prevalence by regions}

The prevalence of cervical HPV infection in physiciancollected sample was, by region, Inner Mongolia (13.0\%), Jiangxi (10.4\%), Hebei (8.7\%), Hubei (8.6\%), Shenzhen (7.1\%) and Guangdong (6.3\%) in descending order. The type-specific prevalence of hr-HPV by regions is presented in Table 1. The overall 5 most frequent genotypes among $995 \mathrm{~h}-\mathrm{HPV}$ positive women were HPV-52 (21.7\%), HPV-16 (19.2\%), HPV-58 (15.0\%), HPV-39 (8.9\%), and HPV-51 (8.1\%). HPV-52 is the most frequent genotype in 4 regions (central and southern mainland China), reaching a high of $40.3 \%$ among the 14 genotypes in Guangdong province. While in the other 2 northern regions HPV-16 remains the most prevalent genotype. HPV-58 is the second most prevalent genotype in Hubei, Jiangxi and Shenzhen and together with HPV-52 and -16 stayed steadily in top 5 genotypes in all 6 regions. Besides the three genotypes above, HPV-39 ranked fourth or fifth in 3 regions, and HPV-68 remained the fifth most prevalent in 2 regions.

\section{Risk of CIN2+/CIN3+ associated with specific HPV genotypes}

The odds ratios for the various hr-HPV genotypes according to regions is shown in Figs. 2, 3, 4, 5, 6, 7, 8. In this multi-center study, the overall odds ratios for CIN2+/CIN3+ in the presence of HPV-16 was 58.6 (95\% CI 39.2-87.5) and, 91.6 (95\%CI 54.3-154.6), respectively. This was much higher than any of the other 13 genotypes. The 4 most frequent genotypes after HPV-16 associated with CIN2+ were: HPV-58 (23.3), HPV-31
(20.7), HPV-33 (16.4), HPV-18 (10.8); and for CIN3+ they were HPV-58 (20.4), HPV-33 (14.0), HPV-56 (12.8), HPV-31 (10.9). By region, HPV-16 remained with the highest odds ratios for CIN2+ in 5 of 6 regions and 3rd highest in Jiangxi just after HPV-31 and HPV-33. But for CIN3+, consistency was seen in all 6 regions with HPV-16 ranking first compared to the other $13 \mathrm{~h}-\mathrm{HPV}$ genotypes. Although HPV-16 imparted great risk of CIN2+/CIN3+ regardless of geographical location, HPV56 showed a higher association than any other genotypes except for HPV-16 with CIN3+ in Inner Mongolia, HPV-31showed the highest odds ratios for CIN2+ and second highest for CIN3+ just after HPV-16 in Jiangxi province, while in Guangdong province HPV-35 held the position of 2nd highest risk after HPV-16.

\section{Discussion}

In May 2018, the Director-General of World Health Organization (WHO), Dr. Tedros Adhanom Ghebreyesus, made a global call to take action to eliminate cervical cancer which is considered one of the greatest threats to women's lives worldwide [12]. To achieve the goal, A modeling study conducted by Kate Simms et al. shows that cervical screening and HPV vaccination are the most two effective methods [13]. Although HPV vaccine could potentially provide up to $90 \%$ protection against cervical cancer according to some research [14], the coverage of vaccination is as low as $1.4 \%$ in low-income and middleincome countries (LMICs). This was far from the modeling requirement of $80-100 \%$ coverage due in large part to the high initial prices of the vaccines [15]. Furthermore,

Table 1 Prevalence of type-specific HPV infection in hr-HPV positive Chinese Han women in physician-collected sample [overall and by region(n: \%)]

\begin{tabular}{|c|c|c|c|c|c|c|c|}
\hline Genotyping & Overall & Inner Mongolia & Hebei Province & Hubei Province & Jangxi Province & Guangdong Province & Shenzhen City \\
\hline HPV-16 & $191 ; 19.2$ & $49 ; 23.0$ & $62 ; 24.4$ & $11 ; 10.9$ & $37 ; 16.5$ & $7 ; 11.3$ & $25 ; 17.7$ \\
\hline HPV-18 & $70 ; 7.0$ & $22 ; 10.3$ & $16 ; 6.3$ & $7 ; 6.9$ & $6 ; 2.7$ & $3 ; 4.8$ & $16 ; 11.3$ \\
\hline HPV-31 & $71 ; 7.1$ & $21 ; 9.9$ & $22 ; 8.7$ & $8 ; 7.9$ & $10 ; 4.5$ & $2 ; 3.2$ & $8 ; 5.7$ \\
\hline HPV-33 & $56 ; 5.6$ & $14 ; 6.6$ & $10 ; 3.9$ & $9 ; 8.9$ & $14 ; 6.3$ & $2 ; 3.2$ & $7 ; 5.0$ \\
\hline HPV-35 & $39 ; 3.9$ & $16 ; 7.5$ & $11 ; 4.3$ & $1 ; 1.0$ & $6 ; 2.7$ & $2 ; 3.2$ & $3 ; 2.1$ \\
\hline HPV-39 & $89 ; 8.9$ & $21 ; 9.9$ & $30 ; 11.8$ & $8 ; 7.9$ & $21 ; 9.4$ & $2 ; 3.2$ & $7 ; 5.0$ \\
\hline HPV-45 & $17 ; 1.7$ & $8 ; 3.8$ & $5 ; 2.0$ & $0 ; 0.0$ & $3 ; 1.3$ & $1 ; 1.6$ & $0 ; 0.0$ \\
\hline HPV-51 & $81 ; 8.1$ & $14 ; 6.6$ & $16 ; 6.3$ & $16 ; 15.8$ & $15 ; 6.7$ & $9 ; 14.5$ & $11 ; 7.8$ \\
\hline HPV-52 & $216 ; 21.7$ & $28 ; 13.1$ & $39 ; 15.4$ & $28 ; 27.7$ & $64 ; 28.6$ & $25 ; 40.3$ & $32 ; 22.7$ \\
\hline HPV-56 & $45 ; 4.5$ & $11 ; 5.2$ & $15 ; 5.9$ & $3 ; 3.0$ & $13 ; 5.8$ & $1 ; 1.6$ & $2 ; 1.4$ \\
\hline HPV-58 & $149 ; 15.0$ & $20 ; 9.4$ & $35 ; 13.8$ & $18 ; 17.8$ & $38 ; 17.0$ & $8 ; 12.9$ & $30 ; 21.3$ \\
\hline HPV-59 & $32 ; 3.2$ & $11 ; 5.2$ & $12 ; 4.7$ & $1 ; 1.0$ & $6 ; 2.7$ & $1 ; 1.6$ & $1 ; 0.7$ \\
\hline HPV-66 & $37 ; 3.7$ & $11 ; 5.2$ & $12 ; 4.7$ & $1 ; 1.0$ & $7 ; 3.1$ & $2 ; 3.2$ & $4 ; 2.8$ \\
\hline HPV-68 & $66 ; 6.6$ & $7 ; 3.3$ & $21 ; 8.3$ & $9 ; 8.9$ & $21 ; 9.4$ & $4 ; 6.5$ & $4 ; 2.8$ \\
\hline Total $^{a}$ & $995 ; 100.0$ & $213 ; 100.0$ & $254 ; 100.0$ & $101 ; 100.0$ & $224 ; 100.0$ & $62 ; 100.0$ & $141 ; 100.0$ \\
\hline
\end{tabular}

${ }^{a}$ On account of multi-infection, the total number of infections may be less than the sum of each HPV genotype 
overall

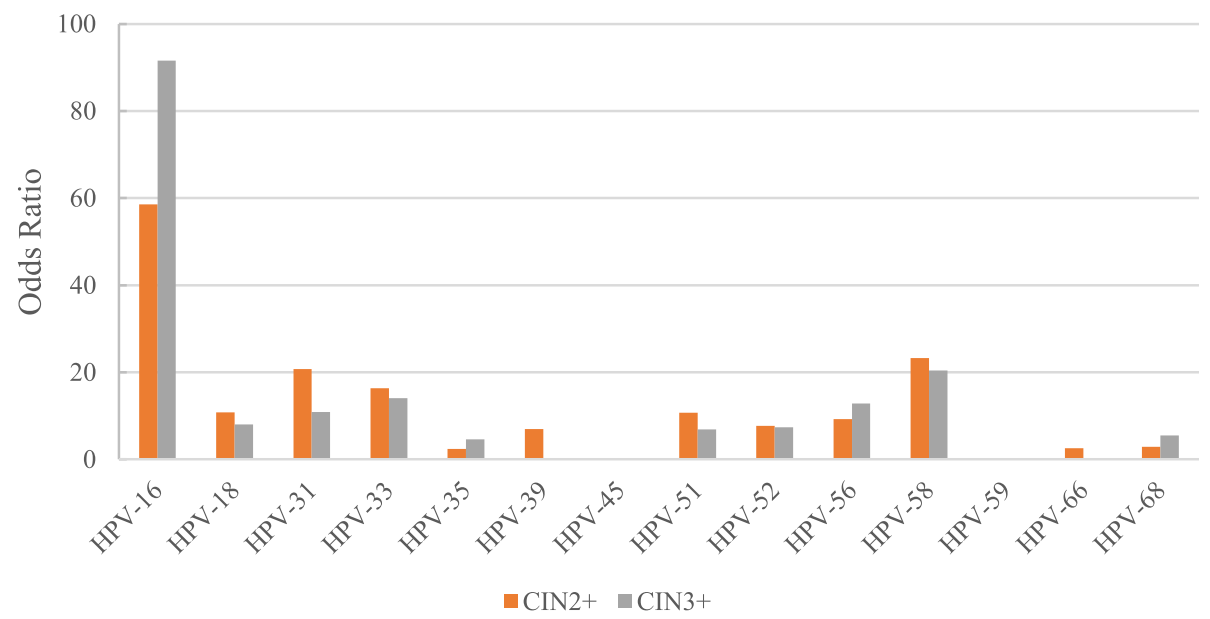

Fig. 2 Risk of CIN2+/CIN3+ according to high-risk Human Papillomavirus (hr-HPV) genotyping, overall

vaccination does not protect against detectable human papillomavirus infections [16]. As a result, screening remains the only feasible preventive option for those already infected and at risk. Compared with the screening based on exfoliative cytology, hr-HPV testing is gradually assuming the more important role in cervical cancer screening. In 2012, the recommended screening strategies were cytology or cytology in combination with hr-HPV testing (cotesting). By 2014 the American Cancer Society (ACS), considered primary hr-HPV screening as an alternative to cytology-based cervical cancer screening [17, 18]. The updated consensus guidelines in 2019 have shifted the management protocols to risk-based algorithms (i.e. "colposcopic referral when immediate risk of having CIN 3+ is $4 \%$ or greater") based primarily on HPV testing with genotyping $[19,20]$. The negative predictive value of HPV testing has been a significant driver of this change since baseline HPV-negative women have a significantly lower cumulative incidence of $\mathrm{CIN} 3+$ at 48 months than cytology-negative women [21, 22]. In addition the effectiveness of self-collection with molecular screening will make it easier in the future to reach women in LMICs who were not well served by cytology-based strategies due to both to human as well as financial constraints [23].

Many studies have shown significant geographic variation in the prevalence of HPV. The estimated HPV prevalence in Sub-Saharan was 24.0\%, Latin America and Caribbean 16.1\%, Eastern Europe 14.2\%, and Southeastern Asia $14.0 \%$ according to a comprehensive meta-analysis involving over 1 million women from 5 continents [24]. In mainland China, various authors have noted significant geographical variation. Li et al. reported Sichuan at 19.9\%

Inner Mongolia

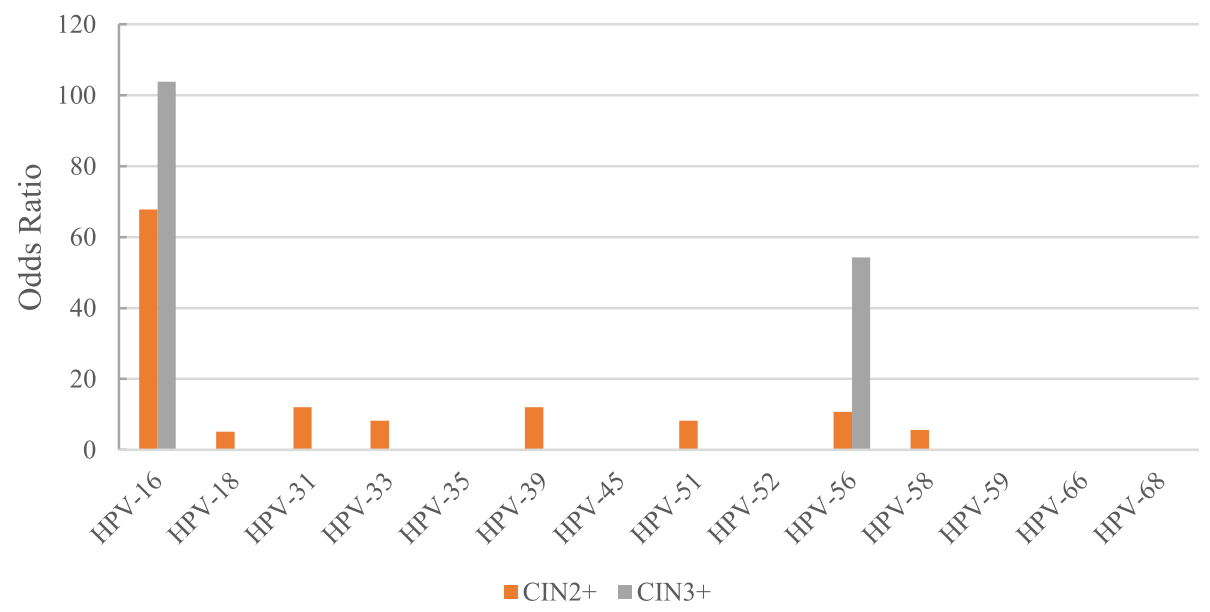

Fig. 3 Risk of CIN2+/CIN3+ according to high-risk Human Papillomavirus (hr-HPV) genotyping, Inner Mongolia 
Hebei Province

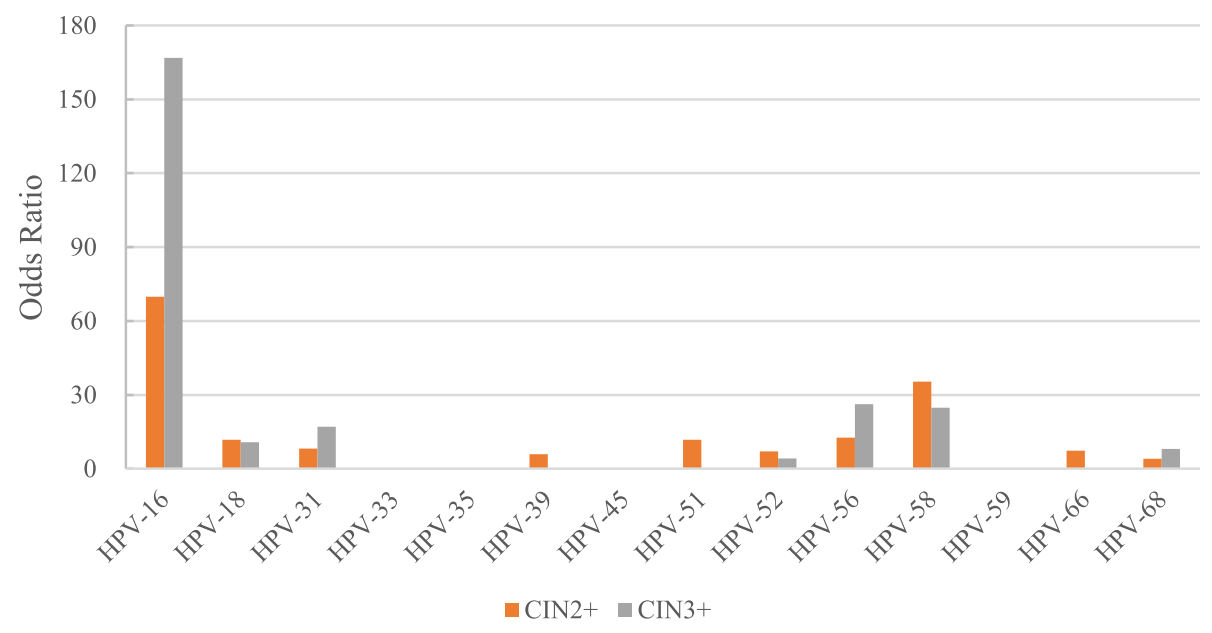

Fig. 4 Risk of CIN2+/CIN3+ according to high-risk Human Papillomavirus (hr-HPV) genotyping, Hebei Province

[25], Shanxi was $26.7 \%$ reported by Cao et al. [26], and Guangdong was $7.3 \%$ reported by Zhao et al. [27] In our study, the overall prevalence of cervical HPV infection is $9.2 \%$ (range 6.3 to $12.9 \%$ by region). This was lower than the $19.0 \%$ prevalence noted by $\mathrm{Li}$ et al. in their systematic review of the epidemiology of hr-HPV infections conducted in mainland China [28].

\section{Inner Mongolia}

Of the 6 regions we studied, Inner Mongolia showed the highest prevalence (13.0\%), similar to that reported by Wang et al. [29], who compared the Han and Mongolian ethnic populations. In CHIMUST, some Mongolian ethnic populations were also recruited. Their details will be fully discussed in a future manuscript.

\section{Guangdong}

The lowest prevalence in our study was in Guangdong (6.3\%), which was less than a half of reported by Zhao et al. in northeastern Guangdong (15.7\%) [30], but just a slightly lower than another population-based survey that enrolled nearly 80,000 women in Guangdong (7.3\%) [27].

\section{Jiangxi}

In an investigation in 11 cities of Jiangxi province [31], the prevalence of hr-HPV infection was 19.5\%, much higher than $10.4 \%$ in our study.

\section{Hubei}

Hubei is in the central mainland China similar to Jiangxi province, the estimated prevalence of hr-HPV was $8.6 \%$

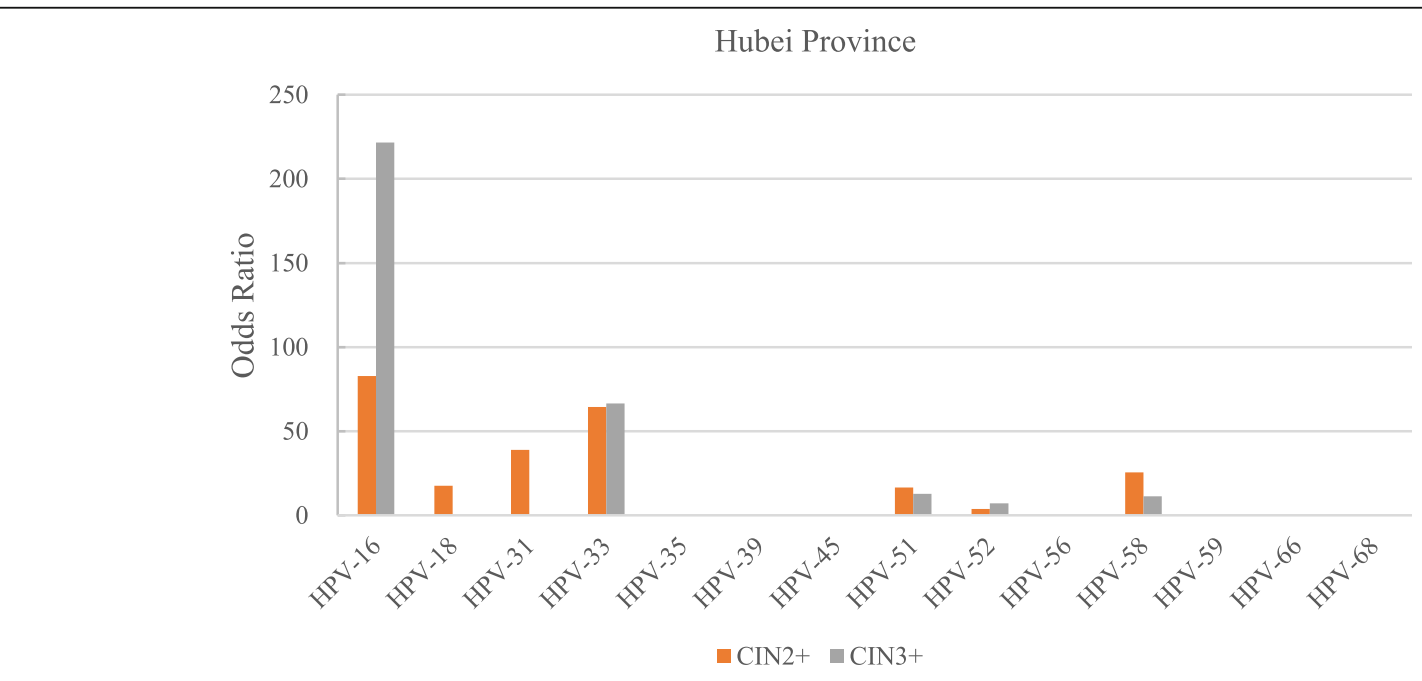

Fig. 5 Risk of CIN2+/CIN3+ according to high-risk Human Papillomavirus (hr-HPV) genotyping, Hubei Province 


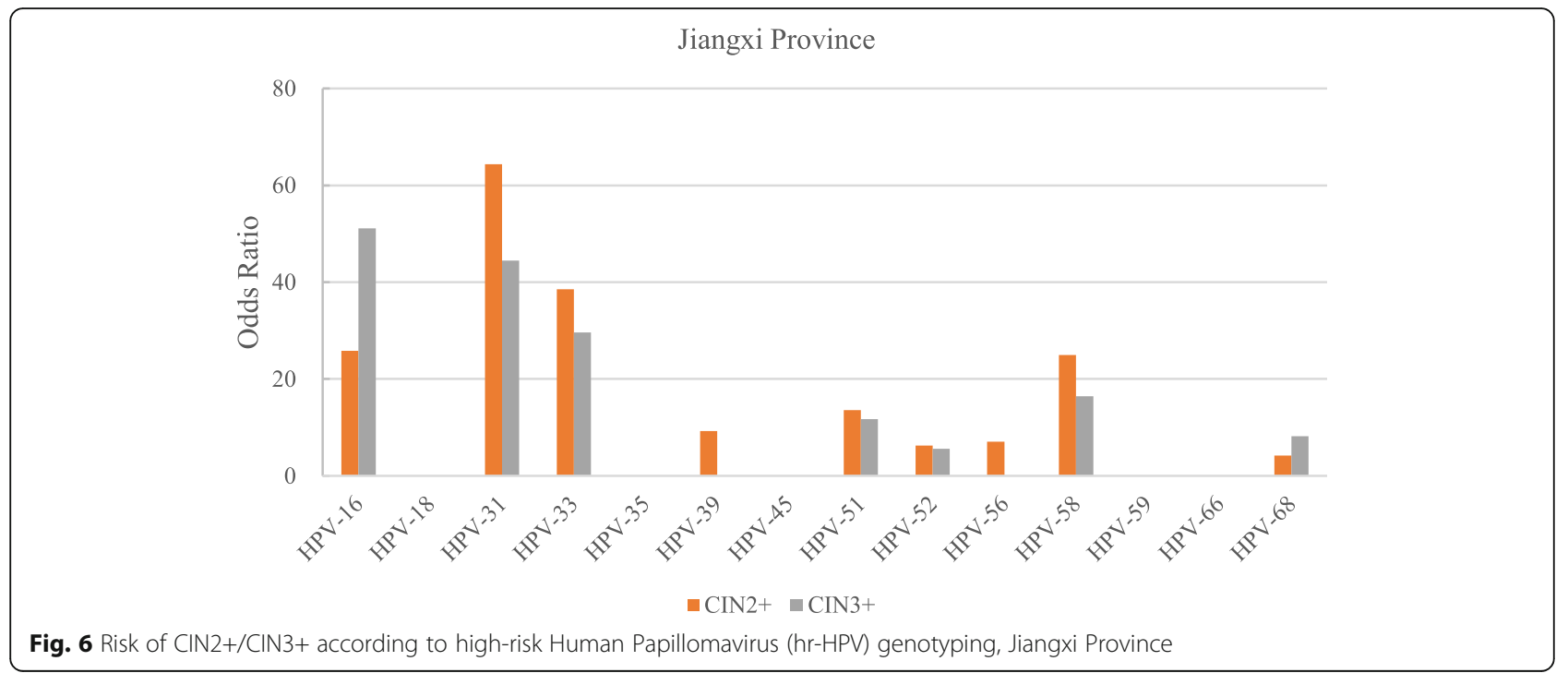

in our study, showed no significant difference with Jiangxi province $\left(x^{2}=2.816, p>0.05\right)$ while the provincial capital, Wuhan, has a higher prevalence (13.9\%) reported by Xiang et al. [32].

\section{Shenzhen}

Shenzhen, located in the very south of Guangdong province, was designated as a national pilot city for comprehensive reform since 1980 . Over the past 40 years, the population in this city has experienced an explosive growth. The permanent population currently exceeds 10 million, almost entirely as a result of migration. The prevalence of hr-HPV infection in general population, according to $\mathrm{Wu}$ et al. reported in 2007, was $13.5 \%$ [33], high than our Shenzhen Pilot Screening results (7.1\%). However, compared with Guangdong province, these two regions in southern mainland China demonstrate a similar prevalence. We assumed that this decreased may relate to the city's rapid industrialization and urbanization, improved medical facilities and access, and the availability of the HPV vaccine. This needs further study.

\section{Hebei}

Hebei province located in northern China had a prevalence of hr-HPV (8.7\%)almost equal to that in Hubei province. In the northern city of Tianjin, which borders on Hebei, hr-HPV infection was reported as (13.5\%) [34]. Generally, there is a decreased trend from north to south in mainland China in the prevalence of HPV infection.

An estimation of population attributable fraction (PAF) of HPV attributable cancer from GLOBOCAN 2012 data showed that HPV-16/18 are responsible globally for $71 \%$ of the cervical cancer [6]. In our study,

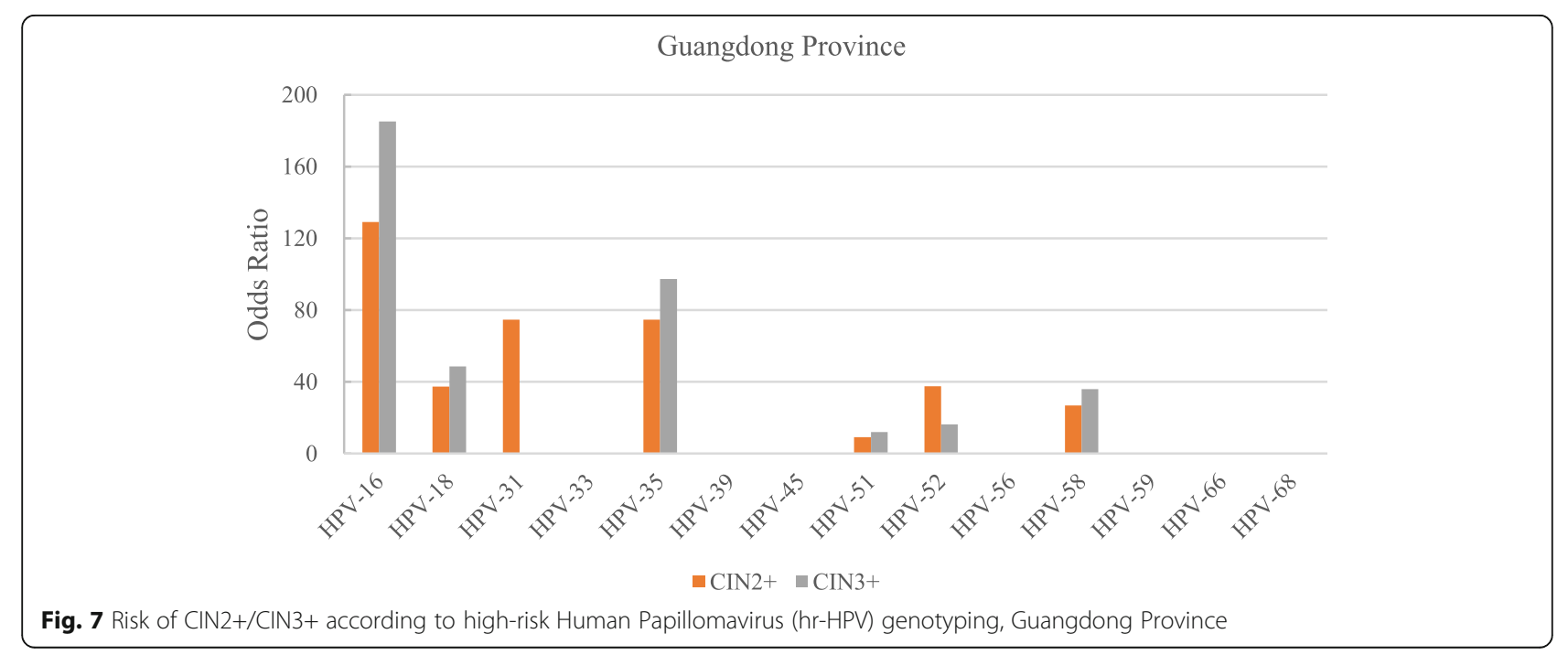


Shenzhen City

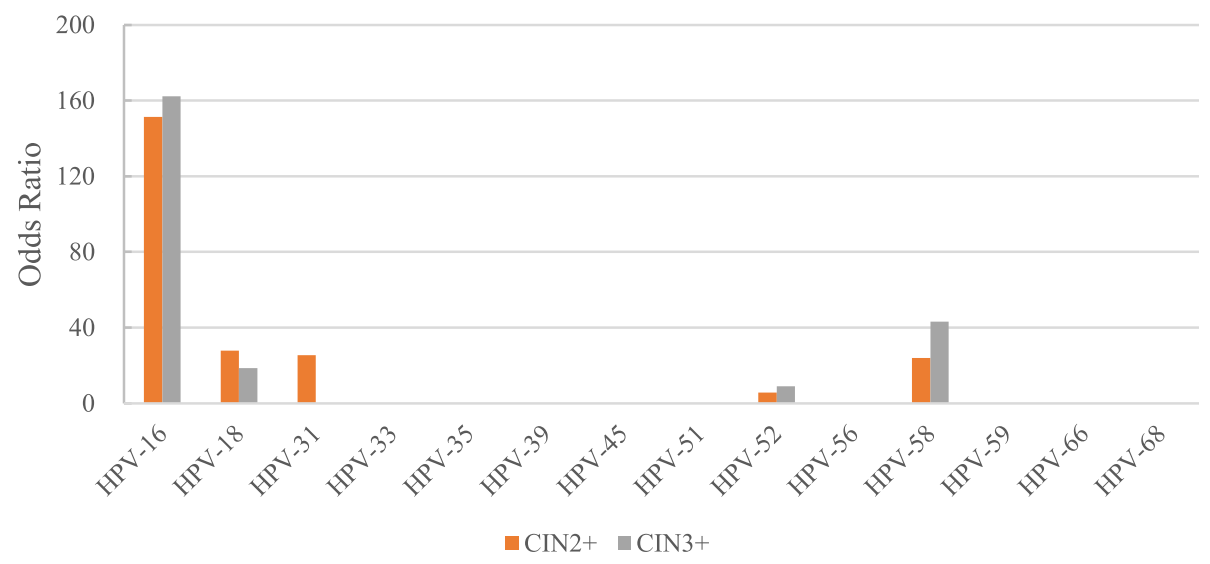

Fig. 8 Risk of CIN2+/CIN3+ according to high-risk Human Papillomavirus (hr-HPV) genotyping, Shenzhen City

HPV-16 remains one of the most dominant genotypes in mainland China just after HPV-52, and is the most frequent genotype in 2 (Inner Mongolia, Hebei) of our 6 regions studied. More importantly, odds ratios for being associated with CIN2+ and CIN3+ of HPV-16 showed a high level of consistency in ranking, being the highest in 5 of 6 regions among all 14 genotypes; and even more dominant for CIN3+. The risk for persistence and progression to cancer precursor lesions varies by HPV type as well as host factors. The specific mechanisms that favor HPV-16 remain a mysterious to be explored. So far, as we know, HPV-16 can be classified into four main evolutionary-derived variant lineages and nine sublineages (A1-4, B1-2, C and D1-3) [35]. By using highthroughput HPV whole-genome sequencing, Mirabello et al. studied the variant lineage risk in over 3200 HPV16-infected women from Kaiser Permanente Northern California (KPNC). Lineage $\mathrm{C}$ showed an increased risk of CIN3 while A4 sub-lineage was associated with an increased risk of adenocarcinoma, suggesting that viral genetic variation may play an important role in carcinogenesis [36]. However, some other HPV whole-genome sequencing studies indicated that strict conservation of HPV-16/ E7, is crucial for viral carcinogenicity [37].

Another meta-analysis on prevalence and attribution of HPV-52 and HPV-58 in cervical neoplasia worldwide indicated that these two genotypes shared a higher prevalence and attribution among cervical intraepithelial neoplasia in Eastern Asia; and the attribution of HPV-58 to invasive cervical cancer was nearly 2 -fold higher than that of HPV-52 [38]. In our study, HPV-52/58 were the two most infectious genotypes, higher than HPV-16 in central and southern mainland China. This is similar to the findings of previous studies [28, 39-41], that HPV$16 / 52 / 58$ were the top 3 genotypes found in mainland China in varying order. It is important to note that although the prevalence of HPV-52 was the highest among 14 genotypes, its carcinogenicity is much lower than HPV-16 and HPV-58, even lower than HPV-31/33/ 18 in most regions. It is reported that HPV-16 was the most common carcinogenic HPV genotype in southwestern China followed by HPV-58 and HPV-33 [42]. But we still believed that HPV-52 infection should not be ignored due to its high frequency and the relatively high risk of cervical intraepithelial neoplasia. $\mathrm{He}$ et al. did a polymorphism analysis of HPV-33/58 in Southwest China and identified some prevalent mutations that could have enhanced viral adaptability to the environment and increased the risk of carcinogenesis [43]. This bio-molecular study also confirmed our observations. A nationwide comprehensive HPV genotyping of invasive cervical cancer in Sweden indicated that the HPV-18 was the second most common genotype after HPV-16, occurring in 1/5 HPV positive women [44]. While in our study, the prevalence of HPV-18 in mainland China was not found to be as high as in western countries, the overall proportion was $7.0 \%$, ranking seventh among the 14 genotypes studied, ranging from $2.7 \%$ in Jiangxi to $11.3 \%$ in Shenzhen. In spite of a relative low proportion in HPV positive women, the odds ratios for CIN2+ and CIN3+ was considerably higher. Chen et al. reported HPV-16/18 were the most frequent HPV genotypes in squamous cell carcinoma (SCC) and cervical adenocarcinoma (CADC), and HPV-18 was more frequent in CADC than in SCC after comparing the distribution of HPV in both histological types [45]. Therefore, the real carcinogenicity of HPV-18 may not be as low as we observed and the bias may be the results of inadequate number of cancer cases in our screening trial, especially glandular lesions.

Our present study has strengths and limitations. This screening trial was a multi-center, cross-sectional, 
population-based study, that enrolled over 10,000 women from northern, central and southern mainland China. Therefore, it allowed a relatively robust analyses of HPV prevalence and distribution under strictly comparable protocols. The colposcopy protocol used in this trial was directed biopsies for all visible lesions, and random biopsies at the squamocolumnar junction in normal quadrants. All colposcopy patients had an endocervical curettage (ECC). We believe this protocol can improve detection of prevalent precancers [8]. Although the total number of participants was large, the sample size from each region is relatively small.

In conclusion, geographical variation existed in the prevalence and distribution of hr-HPV in mainland China. HPV-16/52/58 were the most prevalent genotypes. The present study provided guidance for the refinement of cervical cancer screening strategies and vaccine implementation in China.

\section{Acknowledgements}

None.

\section{Authors' contributions}

All authors helped conduct the project and/or the testing of specimens for HPV and/or data analysis. The author(s) read and approved the final manuscript.

\section{Funding}

This work was supported by Shenzhen High-level Hospital Construction Fund (YBH2019-260), Shenzhen Key Medical Discipline Construction Fund (No. SZXK027) and Sanming Project of Medicine in Shenzhen (No.

SZSM202011016).

\section{Availability of data and materials}

Data available upon reasonable request from corresponding authors. Registered: Chinese Clinical Trials Registry - Chinese Multicenter Cervical Cancer Screening Trial (CHIMUST) ChiCTR-EOC-16008456.

\section{Declarations}

Ethics approval and consent to participate

The study was approved by the Institutional Review Board (IRB) of Peking University Shenzhen Hospital (IRB: PUSH2016001), Shenzhen, China and Cleveland Clinic Institutional Review Board (IRB:15-1549).

\section{Consent for publication}

The authors agree to publication.

\section{Competing interests}

The authors declare no conflict of interest.

\section{Author details}

${ }^{1}$ Department of Obstetrics and Gynecology, Peking University Shenzhen Hospital, No. 1120, Lianhua Road, Shenzhen, Guangdong 518036, PR China. ${ }^{2}$ Shenzhen Key Laboratory on Technology for Early Diagnosis of Major Gynecological Diseases, Shenzhen, Guangdong, PR China. ${ }^{3}$ Preventive Oncology International, Inc. Shaker Heights, USA and Cleveland Clinic, Women's Health Institute, Cleveland, OH, USA. ${ }^{4}$ Sanming Project of Medicine in Shenzhen Peking University Shenzhen Hospital, Shenzhen, Guangdong, PR China.
Received: 1 December 2020 Accepted: 16 March 2021

Published online: 25 March 2021

\section{References}

1. Bray F, Ferlay J, Soerjomataram I, Siegel RL, Torre LA, Jemal A. Global cancer statistics 2018: GLOBOCAN estimates of incidence and mortality worldwide for 36 cancers in 185 countries [published correction appears in CA Cancer J Clin. 2020 Jul;70(4):313]. CA Cancer J Clin. 2018;68(6):394-424. https://doi. org/10.3322/caac.21492.

2. Arbyn M, Weiderpass E, Bruni L, de Sanjosé S, Saraiya M, Ferlay J, Bray F. Estimates of incidence and mortality of cervical cancer in 2018: a worldwide analysis. Lancet Glob Health. 2020;8(2):e191-203. https://doi.org/10.1016/ S2214-109X(19)30482-6.

3. Walboomers JM, Jacobs MV, Manos MM, et al. Human papillomavirus is a necessary cause of invasive cervical cancer worldwide. J Pathol. 1999;189(1): 12-9. https://doi.org/10.1002/(SICI)1096-9896(199909)189:1<12::AID-PATH431 $>3.0 . \mathrm{CO} ; 2-\mathrm{F}$.

4. Harden ME, Munger K. Human papillomavirus molecular biology. Mutat Res Rev Mutat Res. 2017;772:3-12. https://doi.org/10.1016/j.mrrev.2016.07.002.

5. IARC Working Group on the Evaluation of Carcinogenic Risks to Humans. Biological agents. Volume 100 B. A review of human carcinogens. IARC Monogr Eval Carcinog Risks Hum. 2012:100(Pt B):1-441.

6. de Martel C, Plummer M, Vignat J, Franceschi S. Worldwide burden of cancer attributable to HPV by site, country and HPV type. Int J Cancer. 2017; 141(4):664-70. https://doi.org/10.1002/ijc.30716.

7. Serrano B, Brotons M, Bosch FX, Bruni L. Epidemiology and burden of HPVrelated disease. Best Pract Res Clin Obstet Gynaecol. 2018;47:14-26. https:// doi.org/10.1016/j.bpobgyn.2017.08.006.

8. Belinson JL, Pretorius RG. A Standard Protocol for the Colposcopy Exam. J Low Genit Tract Dis. 2016;20(4):e61-2. https://doi.org/10.1097/LGT. 0000000000000239

9. Duan L, Du H, Wang C, et al. The effectiveness of HPV viral load, reflected by Cobas 4800 HPV-Ct values for the triage of HPV-positive women in primary cervical cancer screening: Direct endocervical samples. PLoS One. 2020;15(5):e0232107. Published 2020 May 7. https://doi.org/10.1371/journal. pone.0232107

10. Duan L, Du H, Wang C, et al. The application of BMRT-HPV viral load to secondary screening strategies for cervical cancer. PLoS One. 2020;15(5): e0232117. Published 2020 May 1. https://doi.org/10.1371/journal.pone. 0232117.

11. Yi X, Zou J, Xu J, Liu T, Liu T, Hua S, Xi F, Nie X, Ye L, Luo Y, Xu L, du H, Wu R, Yang L, Liu R, Yang B, Wang J, Belinson JL. Development and validation of a new HPV genotyping assay based on next-generation sequencing. Am J Clin Pathol. 2014;141(6):796-804. https://doi.org/10.1309/AJCP9P2 KJSXEKCJB.

12. WHO Director-General. WHO Director-General calls for all countries to take action to help end the suffering caused by cervical cancer. https://www. who.int/reproductivehealth/call-to-action-elimination-cervical-cancer/en/,202 0-07-10.

13. Simms KT, Steinberg J, Caruana M, Smith MA, Lew JB, Soerjomataram I, Castle PE, Bray F, Canfell K. Impact of scaled up human papillomavirus vaccination and cervical screening and the potential for global elimination of cervical cancer in 181 countries, 2020-99: a modelling study. Lancet Oncol. 2019:20(3):394-407. https://doi.org/10.1016/S1470-2045(18)30836-2.

14. Huh WK, Joura EA, Giuliano AR, Iversen OE, de Andrade RP, Ault KA, Bartholomew D, Cestero RM, Fedrizzi EN, Hirschberg AL, Mayrand MH, RuizSternberg AM, Stapleton JT, Wiley DJ, Ferenczy A, Kurman R, Ronnett BM, Stoler MH, Cuzick J, Garland SM, Kjaer SK, Bautista OM, Haupt R, Moeller E, Ritter M, Roberts CC, Shields C, Luxembourg A. Final efficacy, immunogenicity, and safety analyses of a nine-valent human papillomavirus vaccine in women aged 16-26 years: a randomised, double-blind trial. Lancet. 2017;390(10108):2143-59. https://doi.org/10.1016/S0140-6736(1 7)31821-4.

15. Bruni L, Diaz M, Barrionuevo-Rosas L, Herrero R, Bray F, Bosch FX, de Sanjosé S, Castellsagué X. Global estimates of human papillomavirus vaccination coverage by region and income level: a pooled analysis [published correction appears in Lancet Glob Health. 2017 Jul;5(7): e662]. Lancet Glob Health. 2016;4(7):e453-63. https://doi.org/10.1016/S2214-109X(16)30099-7.

16. Hildesheim A, Gonzalez P, Kreimer AR, et al. Impact of human papillomavirus (HPV) 16 and 18 vaccination on prevalent infections and 
rates of cervical lesions after excisional treatment. Am J Obstet Gynecol. 2016:215(2):212. e1-e15. https://doi.org/10.1016/j.ajog.2016.02.021.

17. Saslow D, Solomon D, Lawson HW, Killackey M, Kulasingam SL, Cain J, Garcia FA, Moriarty AT, Waxman AG, Wilbur DC, Wentzensen N, Downs LS Jr, Spitzer M, Moscicki AB, Franco EL, Stoler MH, Schiffman M, Castle PE, Myers ER, American Cancer Society, American Society for Colposcopy and Cervical Pathology, American Society for Clinical Pathology. American Cancer Society, American Society for Colposcopy and Cervical Pathology, and American Society for Clinical Pathology screening guidelines for the prevention and early detection of cervical cancer. Am J Clin Pathol. 2012; 137(4):516-42. https://doi.org/10.1309/AJCPTGD94EVRSJCG

18. Huh WK, Ault KA, Chelmow D, Davey DD, Goulart RA, Garcia FAR, Kinney WK, Massad LS, Mayeaux EJ, Saslow D, Schiffman M, Wentzensen N, Lawson $H W$, Einstein $M H$. Use of primary high-risk human papillomavirus testing for cervical cancer screening: interim clinical guidance. Gynecol Oncol. 2015; 136(2):178-82. https://doi.org/10.1016/j.ygyno.2014.12.022.

19. Cheung LC, Egemen D, Chen X, Katki HA, Demarco M, Wiser AL, Perkins RB, Guido RS, Wentzensen N, Schiffman M. 2019 ASCCP Risk-Based Management Consensus Guidelines: Methods for Risk Estimation, Recommended Management, and Validation. J Low Genit Tract Dis. 2020; 24(2):90-101. https://doi.org/10.1097/LGT.0000000000000528.

20. Perkins RB, Guido RS, Castle PE, Chelmow D, Einstein MH, Garcia F, Huh WK, Kim JJ, Moscicki AB, Nayar R, Saraiya M, Sawaya GF, Wentzensen N, Schiffman M, 2019 ASCCP Risk-Based Management Consensus Guidelines Committee. 2019 ASCCP Risk-Based Management Consensus Guidelines for Abnormal Cervical Cancer Screening Tests and Cancer Precursors. J Low Genit Tract Dis. 2020;24(2):102-31. https://doi.org/10.1097/LGT. 0000000000000525.

21. Ogilvie GS, van Niekerk D, Krajden M, Smith LW, Cook D, Gondara L, Ceballos K, Quinlan D, Lee M, Martin RE, Gentile L, Peacock S, Stuart GCE, Franco EL, Coldman AJ. Effect of Screening With Primary Cervical HPV Testing vs Cytology Testing on High-grade Cervical Intraepithelial Neoplasia at 48 Months: The HPV FOCAL Randomized Clinical Trial [published correction appears in JAMA. 2018 Dec 4;320(21):2273]. JAMA. 2018;320(1): 43-52. https://doi.org/10.1001/jama.2018.7464.

22. Rebolj M, Rimmer J, Denton $\mathrm{K}$, et al. Primary cervical screening with high risk human papillomavirus testing: observational study. BMJ. 2019;364:240. Published 2019 Feb 6. https://doi.org/10.1136/bmj.l240.

23. Polman NJ, Ebisch RMF, Heideman DAM, Melchers WJG, Bekkers RLM, Molijn AC, Meijer CJLM, Quint WGV, Snijders PJF, Massuger LFAG, van Kemenade FJ, Berkhof J. Performance of human papillomavirus testing on self-collected versus clinician-collected samples for the detection of cervical intraepithelial neoplasia of grade 2 or worse: a randomised, paired screen-positive, noninferiority trial. Lancet Oncol. 2019;20(2):229-38. https://doi.org/10.1016/S14 70-2045(18)30763-0.

24. Bruni L, Diaz M, Castellsagué X, Ferrer E, Bosch FX, de Sanjosé S. Cervical human papillomavirus prevalence in 5 continents: meta-analysis of 1 million women with normal cytological findings. J Infect Dis. 2010;202(12):1789-99. https://doi.org/10.1086/657321.

25. Li B, Wang H, Yang D, Ma J. Prevalence and distribution of cervical human papillomavirus genotypes in women with cytological results from Sichuan province, China. J Med Virol. 2019;91(1):139-45. https://doi.org/10.1002/ jmv.25255.

26. Cao D, Zhang S, Zhang Q, Wei X, Zhao M, Ma Q, Li Y, Wang L, Pei M, Yang $T$, Zhao J, Yang $X$. Prevalence of high-risk human papillomavirus infection among women in Shaanxi province of China: A hospital-based investigation. J Med Virol. 2017;89(7):1281-6. https://doi.org/10.1002/ jmv.24748.

27. Jing L, Zhong X, Zhong Z, Huang W, Liu Y, Yang G, Zhang X, Zou J, Jing C, Wei X. Prevalence of human papillomavirus infection in Guangdong Province, China: a population-based survey of 78,355 women. Sex Transm Dis. 2014;41(12):732-8. https://doi.org/10.1097/OLQ.0000000000000201.

28. Li K, Li Q, Song L, Wang D, Yin R. The distribution and prevalence of human papillomavirus in women in mainland China. Cancer. 2019;125(7):1030-7. https://doi.org/10.1002/cncr.32003.

29. Wang X, Ji Y, Li J, Dong H, Zhu B, Zhou Y, Wang J, Zhou X, Wang Y, Peppelenbosch MP, Pan Q, Ji X, Liu D. Prevalence of human papillomavirus infection in women in the Autonomous Region of Inner Mongolia: A population-based study of a Chinese ethnic minority. J Med Virol. 2018; 90(1):148-56. https://doi.org/10.1002/jmv.24888.
30. Zhao P, Liu S, Zhong Z, et al. Prevalence and genotype distribution of human papillomavirus infection among women in northeastern Guangdong Province of China. BMC Infect Dis. 2018;18(1):204. Published 2018 May 3. https://doi.org/10.1186/s12879-018-3105-x.

31. Zhong TY, Zhou JC, Hu R, Fan XN, Xie XY, Liu ZX, Lin M, Chen YG, Hu XM, Wang WH, Li L, Xiao HP. Prevalence of human papillomavirus infection among 71,435 women in Jiangxi Province, China. J Infect Public Health. 2017;10(6):783-8. https://doi.org/10.1016/j.jiph.2017.01.011.

32. Xiang F, Guan Q, Liu X, Xiao H, Xia Q, Liu X, Sun H, Song X, Zhong Y, Yuan $\mathrm{CH}$, Xiang $\mathrm{Y}$. Distribution characteristics of different human papillomavirus genotypes in women in Wuhan, China. J Clin Lab Anal. 2018;32(8):e22581. https://doi.org/10.1002/jcla.22581.

33. Wu RF, Dai M, Qiao YL, Clifford GM, Liu ZH, Arslan A, Li N, Shi JF, Snijders PJF, Meijer CJLM, Franceschi S. Human papillomavirus infection in women in Shenzhen City, People's Republic of China, a population typical of recent Chinese urbanisation. Int J Cancer. 2007;121(6):1306-11. https://doi.org/10.1 002/ijc.22726.

34. Chen X, Wallin KL, Duan M, Gharizadeh B, Zheng B, Qu P. Prevalence and genotype distribution of cervical human papillomavirus (HPV) among women in urban Tianjin, China. J Med Virol. 2015;87(11):1966-72. https://doi. org/10.1002/jmv.24248.

35. Burk RD, Harari A, Chen Z. Human papillomavirus genome variants. Virology. 2013;445(1-2):232-43. https://doi.org/10.1016/j.virol.2013.07.018.

36. Mirabello L, Yeager M, Cullen M, et al. HPV16 Sublineage Associations With Histology-Specific Cancer Risk Using HPV Whole-Genome Sequences in 3200 Women. J Natl Cancer Inst. 2016;108(9):djw100. Published 2016 Apr 29. https://doi.org/10.1093/jnci/djw100.

37. Mirabello L, Yeager M, Yu K, et al. HPV16 E7 Genetic Conservation Is Critical to Carcinogenesis. Cell. 2017;170(6):1164-1174.e6. https://doi.org/10.1016/j. cell.2017.08.001.

38. Chan PK, Ho WC, Chan MC, et al. Meta-analysis on prevalence and attribution of human papillomavirus types 52 and 58 in cervical neoplasia worldwide. PLoS One. 2014;9(9):e107573. Published 2014 Sep 17. https://doi. org/10.1371/journal.pone.0107573.

39. Zhang C, Cheng W, Liu Q, Guan Q, Zhang Q. Distribution of human papillomavirus infection: a population-based study of cervical samples from Jiangsu Province. Virol J. 2019;16(1):67. Published 2019 May 20. https://doi. org/10.1186/s12985-019-1175-Z.

40. Yuan XW, Li YJ, Qiu Q, Luo ZY, Zhao XF. Prevalence and genotype distribution of human papillomavirus among 9945 women from the Nanhai area of Foshan. BMC Infect Dis. 2019;19(1):71. Published 2019 Jan 18. https:// doi.org/10.1186/s12879-019-3687-y.

41. Liu H, Wei X, Xie Z, Wang X, Gong X, Ke W, Zou H. Cervical human papillomavirus among 19753 women attending gynecological department of a major comprehensive hospital in north Anhui China 2013-2016: Implication for cervical cancer screening and prevention. J Med Virol. 2019; 91(4):698-706. https://doi.org/10.1002/jmv.25365.

42. Long W, Yang Z, Li X, et al. HPV-16, HPV-58, and HPV-33 are the most carcinogenic HPV genotypes in Southwestern China and their viral loads are associated with severity of premalignant lesions in the cervix. Virol J. 2018; 15(1):94. Published 2018 May 25. https://doi.org/10.1186/s12985-018-1003-X.

43. He J, Yang Y, Chen Z, et al. Identification of variants and therapeutic epitopes in HPV-33/HPV-58 E6 and E7 in Southwest China. Virol J. 2019; 16(1):72. Published 2019 May 28. https://doi.org/10.1186/s12985-019-1168-y.

44. Lagheden C, Eklund C, Lamin H, Kleppe SN, Lei J, Elfström KM, Sundström K, Andrae B, Sparén P, Dillner J. Nationwide comprehensive human papillomavirus (HPV) genotyping of invasive cervical cancer. $\mathrm{Br} J$ Cancer. 2018;118(10):1377-81. https://doi.org/10.1038/s41416-018-0053-6.

45. Chen W, Sun $H$, Molijn A, Zeng L, Kang L, Jenkins D, Zhang S, Cui J, Wu Z, Pirog E, Shen G, Zhang X, Qiao Y, Chinese HPV Typing Group. The Variable Characteristics of Human Papillomavirus in Squamous Cell Carcinoma and Adenocarcinoma of Cervix in China. J Low Genit Tract Dis. 2018;22(4):35561. https://doi.org/10.1097/LGT.0000000000000408.

\section{Publisher's Note}

Springer Nature remains neutral with regard to jurisdictional claims in published maps and institutional affiliations. 